\title{
ON THE CANONICAL RINGS OF SOME HORIKAWA SURFACES. PART I
}

\author{
VALENTIN ILIEV
}

\begin{abstract}
This paper is devoted to finding necessary and sufficient conditions for a graded ring to be the canonical ring of a minimal surface of general type with $K^{2}=2 p_{g}-3, p_{g} \geq 3$, and such that its canonical linear system has one base point.
\end{abstract}

Introduction. The minimal surfaces $S$ of general type with $K^{2}=2 p_{g}-3$, $p_{g} \geq 3$, defined over the field of complex numbers $\mathbf{C}$ were studied by $\mathbf{E}$. Horikawa in [4 and 5]. Because of the fact that these surfaces are regular (cf. [1, Theorem 10]) and because of the vanishing of $H^{1}(S, \mathscr{O}(m K))$ for all $m \geq 2$ (cf. [7, Theorem 5]) the restriction maps $H^{0}(S, \mathscr{O}(m K)) \rightarrow H^{0}\left(K, \mathscr{O}\left(\left.K\right|_{K}\right)\right)$ are surjective for all $m \geq 0$ and it becomes possible to use the hyperplane section principle. This is a very suggestive idea proposed by Miles Reid (cf. also [2]) and we make use of it in giving an algebraic treatment of Horikawa's papers.

Throughout the rest of this paper we suppose that the canonical linear system $|K|$ has one base point. This is not a restriction when $p_{g} \geq 5$ (cf. $\left.[\mathbf{5}, \S 1]\right)$.

$\S 1$ and Appendix A contain mostly known results. They are included because we cannot find references appropriate for our purposes. In $\S 2$ we describe a ring which is "the trace" of the canonical ring $R$ on a generic member of $|K|$. $\S 3$ contains a presentation of the canonical ring $R$ by generators and relations. $\S \S 4$ and 5 are devoted to finding necessary conditions which a posteriori are shown to be also sufficient for a graded ring $R$ to be the canonical ring of a minimal surface of general type with $p_{g}=n+1, K^{2}=2 n-1, n \geq 2$, and such that the canonical linear system $|K|$ has one base point.

This work has two main points in common with [4 and 5]: $\S 1$, where we give Horikawa's Lemma 2 from [4] and his description of the canonical image from [5] in the present context, and subsections 5.1 and 5.2, where we use Lemmas 5, 6 from [4] and Lemma 1.2 from [5].

The cases when $p_{g}=3, K^{2}=3$ or $p_{g}=4, K^{2}=5$ are studied in [6 and 2] respectively. With our present approach we obtain these results as special cases.

\section{Known results.}

1.1. Let $S$ be a minimal surface of general type with $K^{2}=2 p_{g}-3, p_{g} \geq 3$. We assume that the canonical linear system $|K|$ of $S$ has one base point $P$. Following Horikawa [4, Lemma 2] let $\pi: S^{\prime} \rightarrow S$ be the quadratic transformation with center $P$ and exceptional curve $E$. Then $\left|\pi^{*} K\right|=|L|+E$ where the linear system $|L|$ is without base points, hence its generic member $L$ is an irreducible and nonsingular

Received by the editors November 18, 1985 and, in revised form, June 23, 1987.

1980 Mathematics Subject Classification (1985 Revision). Primary 14J25.

Key words and phrases. Surface of general type, canonical ring. 
curve. Set $p_{g}=n+1$. Then $n \geq 2, K^{2}=2 n-1, L^{2}=2 n-2$, the genus of $L$ is $2 n$ and $(E, L)=1$. In particular, the generic member $C$ of $|K|$ is smooth at $P$, hence $\pi$ induces an isomorphism between $L$ and $C$. We have $\operatorname{dim} H^{0}(S, \mathscr{O}(L-E))=$ $\operatorname{dim} H^{0}(S, \mathscr{O}(K-2 P))=n-1$ and the short exact sequence

$$
0 \rightarrow \mathscr{O}_{S^{\prime}}(L-E) \rightarrow \mathscr{O}_{S^{\prime}}(L) \rightarrow \mathscr{O}_{E}(1) \rightarrow 0
$$

shows that the linear system $|L|$ cuts out the whole linear system of degree 1 on $E \cong \mathrm{P}^{1}$. We denote by $F_{L}$ the regular map $S^{\prime} \rightarrow \mathbf{P}^{n}$ defined by $|L|$ and let $W$ be its image. It is clear that $F_{L}$ induces an isomorphism $E \stackrel{\cong}{\rightrightarrows} l$ where $l=F_{L}(E)$. The short exact sequence

$$
0 \rightarrow \mathscr{O}_{S^{\prime}} \rightarrow \mathscr{O}_{S^{\prime}}(L) \rightarrow \mathscr{O}_{L}\left(\left.L\right|_{L}\right) \rightarrow 0
$$

gives that $\operatorname{dim} H^{0}\left(L, \mathscr{O}_{L}\left(\left.L\right|_{L}\right)\right)=n$ and this fact together with $\left.\operatorname{deg} L\right|_{L}=L^{2}=$ $2(n-1)$ implies (by the theorem of Clifford) that the curve $L$ is hyperelliptic and $\left.L\right|_{L}=(n-1) g_{2}^{1}$, where $g_{2}^{1}$ is the unique linear system of dimension 1 and degree 2 on $L$. Therefore the $F_{L}$-image $H$ of $L$ is a rational normal curve of degree $n-1$ in $\mathbf{P}^{n-1} \subset \mathbf{P}^{n}$ and this is the generic hyperplane section of $W$. Because of $\operatorname{deg} W=n-1$ we obtain that $W$ is a rational normal scroll in $\mathbf{P}^{n}: W=W_{c, d}$, $c \geq 0, d \geq 0,2 c+d=n-1$ (cf. [3, Chapter 4 and 5, $\S 1]$ ), and $\operatorname{deg} F_{L}=2$. We set

$$
\begin{aligned}
E_{0}= & \text { a zero section of } W_{c, d}, \\
E_{\infty}= & \text { the directrix of } W_{c, d} \text { in case } c>0, \text { or the vertex of } \\
& \text { the cone } W_{0, n-1}, \\
f= & \text { the generic fibre of } W_{c, d} .
\end{aligned}
$$

Because of $(L, E)=1$ we obtain $(H, l)=1$ so that $l$ is a line in $\mathbf{P}^{n}$ on $W_{c, d}$. When $c>0$ it is obvious that $(l, f)=\varepsilon$, where $\varepsilon=0$ or 1 . In case $\varepsilon=0$ (let us call it Case A) $l$ is a fibre of $W_{c, d}$. In the second case $\varepsilon=1$ (let us call it Case B) it is clear that $l=E_{\infty}$ and $c=1$. We will refer to the case when $W_{c, d}$ is a cone $(c=0)$ as Case $\mathrm{C}$. Then $l$ is a ruling of the cone.

1.2. Let $W_{c, d}$ be a rational normal scroll in $\mathbf{P}^{n}$ and let $\left(z_{0}, z_{1}\right)$ be a basis of $H^{0}\left(E_{0}, \mathscr{O}(1)\right)$. It is easy to see that there exists a coordinate system $(x ; y)=$ $\left(x_{0}, \ldots, x_{c} ; y_{0}, \ldots, y_{c+d}\right)$ in $\mathbf{P}^{n}$ such that the fibre $f_{\left(z_{0}, z_{1}\right)}$ of $W_{c, d}$ through $\left(z_{0}, z_{1}\right) \in$ $E_{0}$ is the line which joins the point $\left(z_{1}^{c}, z_{0} z_{1}^{c-1}, \ldots, z_{0}^{c} ; 0, \ldots, 0\right) \in E_{\infty}$ to the point $\left(0, \ldots, 0 ; z_{1}^{c+d}, z_{0} z_{1}^{c+d-1}, \ldots, z_{0}^{c+d}\right) \in E_{0}$. The surface $W_{c, d}$ is the locus of the points in $\mathbf{P}^{n}$ which have the form

$$
\left(\alpha z_{1}^{c}, \alpha z_{0} z_{1}^{c-1}, \ldots, \alpha z_{0}^{c} ; \beta z_{1}^{c+d}, \beta z_{0} z_{1}^{c+d-1}, \ldots, \beta z_{0}^{c+d}\right)
$$$$
(\alpha, \beta) \neq(0,0)
$$

Hence $W_{c, d}$ has the following equations in $\mathbf{P}^{n}$ :

$$
\begin{array}{cc}
a_{i j}=x_{i} x_{j+1}-x_{i+1} x_{j}=0, & 0 \leq i<j \leq c-1, \\
b_{l m}=y_{l} y_{m+1}-y_{l+1} y_{m}=0, & 0 \leq l<m \leq c+d-1, \\
c_{i m}=x_{i} y_{m+1}-x_{i+1} y_{m}=0, & 0 \leq i \leq c-1,0 \leq m \leq c+d-1 .
\end{array}
$$

Let $H=(h(x, y)=0)$ be a hyperplane section of $W_{c, d}$, where

$$
h(x, y)=a(x)+b(y), \quad a(x)=\sum_{i=0}^{c} a_{i} x_{i}, b(y)=\sum_{j=0}^{c+d} b_{j} y_{j} .
$$


We set

$$
\begin{gathered}
a\left(z_{0}, z_{1}\right)=\sum_{i=0}^{c} a_{i} z_{0}^{i} z_{1}^{c-i}, \quad b\left(z_{0}, z_{1}\right)=\sum_{j=0}^{c+d} b_{j} z_{0}^{j} z_{1}^{c+d-j} \\
d\left(z_{0}, z_{1}\right)=\text { the greatest common divisor of } a\left(z_{0}, z_{1}\right) \text { and } b\left(z_{0}, z_{1}\right), \\
a_{1}\left(z_{0}, z_{1}\right)=a\left(z_{0}, z_{1}\right) / d\left(z_{0}, z_{1}\right), \quad b_{1}\left(z_{0}, z_{1}\right)=b\left(z_{0}, z_{1}\right) / d\left(z_{0}, z_{1}\right) .
\end{gathered}
$$

An immediate consequence of form (1.2.1) of the generic point of $W_{c, d}$ is the following.

LEMMA 1.2.6. If $d\left(z_{0}, z_{1}\right)=\left(z_{1}^{(1)} z_{0}-z_{0}^{(1)} z_{1}\right) \cdots\left(z_{1}^{(s)} z_{0}-z_{0}^{(s)} z_{1}\right), s \geq 0$, then

$$
H=\left\{\begin{array}{l}
H_{1}+f_{\left(z_{0}^{(1)}, z_{1}^{(1)}\right)}+\cdots+f_{\left(z_{0}^{(s)}, z_{1}^{(s)}\right)}, \quad c>0, \\
H_{1}, \quad c=0, E_{\infty} \notin H, \\
f_{\left(z_{0}^{(1)}, z_{1}^{(1)}\right)}+\cdots+f_{\left(z_{0}^{(s)}, z_{1}^{(s)}\right)}, \quad c=0, E_{\infty} \in H,
\end{array}\right.
$$

where $H_{1}$ is the rational curve on $W_{c, d}$ with parametric representation

$$
\begin{aligned}
& x_{i}=-b_{1}\left(z_{0}, z_{1}\right) z_{0}^{i} z_{1}^{c-i}, \quad i=0, \ldots, c, \\
& y_{j}=a_{1}\left(z_{0}, z_{1}\right) z_{0}^{j} z_{1}^{c+d-j}, \quad j=0, \ldots, c+d .
\end{aligned}
$$

COROLLARY 1.2.7. (a) The fibre $f_{\left(z_{0}, z_{1}\right)}$ is a component of $H$ if and only if $\left(z_{0}, z_{1}\right)$ is a common zero of the polynomials $a\left(z_{0}, z_{1}\right)$ and $b\left(z_{0}, z_{1}\right)$.

(b) The directrix (the vertex) $E_{\infty}$ is a component of $H$ (belongs to $H$ ) if and only if $a\left(z_{0}, z_{1}\right)=0$.

(c) The hyperplane section $H$ is irreducible (and hence a smooth curve $\cong \mathbf{P}^{1}$ ) if and only if $R(a, b) \neq 0$ where $R(a, b)$ is the resultant of the polynomials $a\left(z_{0}, z_{1}\right)$ and $b\left(z_{0}, z_{1}\right)$. In this case $H$ has a parametric representation

$$
\begin{aligned}
x_{i} & =-\sum_{j=0}^{c+d} b_{j} z_{0}^{i+j} z_{1}^{n-1-i-j}, \quad i=0, \ldots, c, \\
y_{j} & =\sum_{i=0}^{c} a_{i} z_{0}^{i+j} z_{1}^{n-1-i-j}, \quad j=0, \ldots, c+d .
\end{aligned}
$$

1.3. Let $H=(h(x, y)=0)$ be an irreducible hyperplane section of $W_{c, d}$. Formulae (1.2.8) define an isomorphism $\mathbf{P}^{1} \stackrel{\cong}{\rightrightarrows} H$ which can be decomposed into a Veronese embedding $\mathbf{P}^{1} \rightarrow \mathbf{P}^{n-1}, \bar{x}_{k}=z_{0}^{k-1} z_{1}^{n-k}, k=1, \ldots, n$, followed by the natural embedding $\mathbf{P}^{n-1}=\left(\bar{x}_{0}=0\right) \subset \mathbf{P}^{n}$ and by the automorphism $\left(\bar{x}_{k}\right) \rightarrow(x, y)$ of $\mathbf{P}^{n}$ which is given in Cases $\mathrm{A}$ and $\mathrm{C}$, under the assumption $b_{0} \neq 0$, by the matrix (1.3.1)

$$
\left(\begin{array}{cccccccccc}
0 & -b_{0} & -b_{1} & -b_{2} & \cdots & -b_{c+d} & & 0 & \cdots & 0 \\
0 & 0 & -b_{0} & -b_{1} & \cdots & -b_{c+d-1} & & -b_{c+d} & \cdots & 0 \\
\vdots & \vdots & \vdots & \vdots & & & & & & \vdots \\
0 & 0 & 0 & 0 & \cdots & -b_{0} & & -b_{1} & \cdots & -b_{c+d} \\
\frac{1}{b_{0} R(b, a)} & a_{0} & a_{1} & a_{2} & \cdots & & a_{c} & 0 & \cdots & 0 \\
0 & 0 & a_{0} & a_{1} & \cdots & & a_{c-1} & a_{c} & \cdots & 0 \\
\vdots & \vdots & \vdots & \vdots & & & & & & \vdots \\
0 & 0 & 0 & 0 & \cdots & a_{0} & & a_{1} & \cdots & a_{c}
\end{array}\right)
$$


and in Case B (cf. also Remark 2.1.1) by the matrix

$$
\left(\begin{array}{ccccccc}
0 & -b_{0} & -b_{1} & -b_{2} & \cdots & -b_{1+d} & 0 \\
\frac{1}{a_{1} R(b, a)} & 0 & -b_{0} & -b_{1} & \cdots & -b_{d} & -b_{1+d} \\
0 & a_{0} & a_{1} & 0 & \cdots & 0 & 0 \\
0 & 0 & a_{0} & a_{1} & \cdots & 0 & 0 \\
\vdots & \vdots & \vdots & \vdots & & \vdots & \vdots \\
0 & 0 & 0 & 0 & \cdots & a_{0} & a_{1}
\end{array}\right)
$$

Note that in all cases the determinant is 1 . The inverse automorphism has the form

$$
\begin{aligned}
& \bar{x}_{0}=R(b, a) h(x, y), \\
& \bar{x}_{k}=h_{k}(x, y), \quad k=1, \ldots, n,
\end{aligned}
$$

where $h_{k}(x, y)=c_{k}(x)+d_{k}(y)$ are linear forms such that after expressing $x$ and $y$ by means of (1.2.8), one gets

$$
z_{0}^{k-1} z_{1}^{n-k}=h_{k}(x, y)=-b\left(z_{0}, z_{1}\right) c_{k}\left(z_{0}, z_{1}\right)+a\left(z_{0}, z_{1}\right) d_{k}\left(z_{0}, z_{1}\right)
$$

for $k=1, \ldots, n$. Our choice of the first column in the matrices (1.3.1) and (1.3.2) means that $d_{k}(0,1)$ is zero for $k=1, \ldots, n$ in Cases $\mathrm{A}$ and $\mathrm{C}$, and $c_{k}(1,0)$ is zero for $k=1, \ldots, n$ in Case B (cf. Appendix A, A.1).

\section{Rings on a hyperelliptic curve.}

2.1. Let $H=(h(x, y)=0)$ be an irreducible hyperplane section of $W=W_{c, d}$ such that the curve $L=F_{L}^{*}(H)$ is irreducible and nonsingular. Let us denote the point $L \cap E$ by $P^{\prime}$ and a generator of the C-linear space $H^{0}\left(S^{\prime}, \mathscr{O}(E)\right)$ by $e$. We have an isomorphism $H^{0}\left(S^{\prime}, \mathscr{O}(E)\right) \rightarrow H^{0}\left(L, \mathscr{O}\left(P^{\prime}\right)\right)$ which is induced by the restriction map. Hence $H^{0}\left(L, \mathscr{O}\left(P^{\prime}\right)\right)=\mathrm{C} s$ where $s$ is the restriction of section $e$ on $L$. Let $P_{h}$ be the intersection point of the line $l$ with $H$. We can suppose $\left(z_{0}\right)=P_{h}$ on the curve $H$, hence we have $z_{0}=s^{2}$ on the curve $L$ and $P^{\prime}$ is a branch point of this hyperelliptic curve. Setting $z_{1}=t$ we have $H^{0}\left(L, \mathscr{O}\left(2 P^{\prime}\right)\right)=\mathbf{C} s^{2} \oplus \mathbf{C} t$.

REMARK 2.1.1. In Case B equality $\left(z_{0}\right)=P_{h}$ implies $a_{0}=0$ and therefore $a_{1} \neq 0(h(x, y)$ is given by expression $(1.2 .5)$ and $R(a, b) \neq 0$, cf. Corollary 1.2.7(c)). If $C \in|K|$ is such that $\pi^{*}(C)=L+E$, then we can transfer sections $s$ and $t$ on the hyperelliptic curve $C$. The base point $P$ of $|K|$ is a branch point of $C$ and $H^{0}(C, \mathscr{O}(P))=\mathbf{C} s, H^{0}(C, \mathscr{O}(2 P))=\mathbf{C} s^{2} \oplus \mathbf{C} t$. The inverse images $x_{i}^{\prime}=F_{L}^{*}\left(x_{i}\right)$, $y_{j}^{\prime}=F_{L}^{*}\left(y_{j}\right)$ of the homogeneous coordinates in $\mathbf{P}^{n}$ form a basis of $H^{0}\left(S^{\prime}, \mathscr{O}(L)\right)$. Let $x_{i}, y_{j}$ be the sections in $H^{0}(C, \mathscr{O}(K))$ for which $\pi^{*}\left(x_{i}\right)=x_{i}^{\prime} e, \pi^{*}\left(y_{j}\right)=y_{j}^{\prime} e$. These sections span the C-linear space $H^{0}(S, \mathscr{O}(K))$ and define the canonical map $F_{K}$. We can rewrite (1.2.8) and (1.3.3) on the curve $C$ in the form

$$
\begin{gathered}
x_{i}=-s b\left(s^{2}, t\right) s^{2 i} t^{c-i}, \quad i=0, \ldots, c, \\
y_{j}=s a\left(s^{2}, t\right) s^{2 j} t^{c+d-j}, \quad j=0, \ldots, c+d, \\
s^{2 k-1} t^{n-k}=h_{k}(x, y), \quad k=1, \ldots, n .
\end{gathered}
$$

In particular, $s^{2 n-1}=h_{n}(x, y)$, hence $\left.K\right|_{C} \sim(2 n-1) P$. Denote

$$
R=\bigoplus_{m \geq 0} H^{0}(S, \mathscr{O}(m K)), \quad R_{C}=\bigoplus_{m \geq 0} H^{0}(C, \mathscr{O}(m(2 n-1) P)) .
$$


Since the surface $S$ is regular, the restriction map $R \rightarrow R_{C}$ is surjective and it is clear that the description of the ring $R_{C}$ will be helpful in order to describe the canonical ring $R$.

2.2. Let $C$ be a hyperelliptic curve of genus $g$ and let $P$ be its branch point. We set $A=\bigoplus_{m \geq 0} H^{0}(C, \mathscr{O}(m P))$.

LEMMA 2.2.1. The graded ring $A$ can be presented as $\mathbf{C}[s, t, u] /\left(F_{4 g+2}\right)$, where $\operatorname{deg} s=1, \operatorname{deg} t=2, \operatorname{deg} u=2 g+1, F_{4 g+2}=u^{2}-f_{2 g+1}\left(s^{2}, t\right)$ and the zeroes of the polynomial $f_{2 g+1}\left(z_{0}, z_{1}\right)$ are all the branch points of the unique 2-1 map $C \rightarrow \mathbf{P}^{1}$, except the branch point $(0,1) \in \mathbf{P}^{1}$. Moreover, the sections $s^{m}, s^{m-2} t, \ldots$ span the $(+)$ eigenspace, and the sections $s^{m-2 g-1} u, s^{m-2 g-3} t u, \ldots, m \geq 2 g+1$, span the $(-)$ eigenspace in degree $m$ of the hyperelliptic involution $i$ of $C$.

ProOF. One can use the same arguments as in [6, Lemma 2].

REMARK 2.2.2. The polynomial $f_{2 g+1}\left(z_{0}, z_{1}\right)=\sum_{\nu=0}^{2 g+1} e_{\nu} z_{0}^{\nu} z_{1}^{2 g+1-\nu}$ has simple roots and $e_{0} \neq 0$.

Suppose $g=2 n, n \geq 2$, and set

$$
\begin{aligned}
& h_{k}=s^{2 k-1} t^{n-k}, \quad k=1, \ldots, n, \\
& z=t^{2 n-1}, \\
& v_{p}=s^{2 n-2 p-2} t^{p-1} u, \quad p=1, \ldots, n-1 .
\end{aligned}
$$

Let

$$
a\left(z_{0}, z_{1}\right)=\sum_{i=0}^{c} a_{i} z_{0}^{i} z_{1}^{c-i} \quad \text { and } \quad b\left(z_{0}, z_{1}\right)=\sum_{j=0}^{c+d} b_{j} z_{0}^{j} z_{1}^{c+d-j}
$$

be mutually prime polynomials, $2 c+d=n-1$, and let $x_{i}, y_{j}$ be the sections in $H^{0}(C, \mathscr{O}((2 n-1) P))$ given by formulae (2.1.2). We can express $h_{k}$ in terms of $x$ and $y$ by formulae (2.1.3) and all the relations among $x_{i}$ and $y_{j}$ are given by $h(x, y)=0$ and equations (1.2.2), (1.2.3), and (1.2.4).

The products $v_{p} v_{q}, 1 \leq p \leq q \leq n-1$, are $i$-invariant, hence there exist $2 n-$ 3 homogeneous degree 3 polynomials $\varphi_{11}, \varphi_{12}, \varphi_{22}, \ldots, \varphi_{n-2, n-1}, \varphi_{n-1, n-1}$, such that

$$
\begin{aligned}
& f_{p q}=v_{p} v_{q}-\varphi_{[(p+q) / 2][(p+q+1) / 2]}\left(h_{1}^{2}, h_{1} h_{2}, \ldots, h_{n-1} h_{n}, h_{n}^{2}, z\right)=0, \\
& \varphi_{[(p+q) / 2][(p+q+1 / 2)]}\left(z_{0} z_{1}^{2 n-2}, z_{0}^{2} z_{1}^{2 n-3}, \ldots, z_{0}^{2 n-1}, z_{1}^{2 n-1}\right) \\
& \quad=z_{0}^{2 n-p-q-2} z_{1}^{p+q-2} f_{4 n+1}\left(z_{0}, z_{1}\right),
\end{aligned}
$$

for all $p, q$ with $1 \leq p \leq q \leq n-1$. Using (2.2.3) we get the remaining relations among the $x_{i}, y_{j}, z, v_{p}$ :

$$
d_{k}=h_{k} h_{1}^{2}-h_{k+1} z=0, \quad k=1, \ldots, n-1,
$$

$$
\begin{gathered}
g_{k s p q}=h_{k} v_{p}-h_{s} v_{q}=0, \quad 1 \leq p<q \leq n-1,1 \leq k<s \leq n, s+p=k+q, \\
(2.2 .8) \quad h_{p q}=z v_{p}-h_{[(q-p+1) / 2]} h_{[(q-p) / 2]+1} v_{q}=0, \quad 1 \leq p<q \leq n-1 .
\end{gathered}
$$

Let us denote $v=\left(v, \ldots, v_{n-1}\right)$. We have proved the theorem below which characterizes the ring $R_{C}$. 
THEOREM 2.2.9. There exists a natural isomorphism of graded rings $R_{C} \cong$ $\mathbf{C}[x, y, z, v] / I_{h}$, where $\operatorname{deg} x_{i}=\operatorname{deg} y_{j}=1, \operatorname{deg} z=2, \operatorname{deg} v_{p}=3$, and $I_{h}$ is the ideal generated by the polynomials $h(c f .(1.2 .5))$ of degree $1, a_{i j}, b_{l m}, c_{i m}(c f$. (1.2.2), (1.2.3), (1.2.4)) of degree $2, d_{k}(c f .(2.2 .6))$ of degree $3, g_{k s p q}(c f .(2.2 .7))$ of degree $4, h_{p q}(c f .(2.2 .8))$ of degree $5, f_{p q}(c f .(2.2 .4))$ of degree 6.

REMARK 2.2.10. The syzygies

$$
\begin{gathered}
v_{r} g_{k s p q}-h_{k} f_{p r}+h_{s} f_{q r}=0, \quad \begin{array}{r}
1 \leq p<q \leq n-1,1 \leq k<s \leq n, \\
s+p=k+q, 1 \leq r \leq n-1,
\end{array} \\
v_{r} h_{p q}-z f_{p r}+h_{[(q-p+1) / 2]} h_{[(q-p) / 2]+1} f_{q r}=0, \\
\quad \begin{array}{l}
1 \leq p<q \leq n-1, \\
1 \leq r \leq n-1,
\end{array}
\end{gathered}
$$

and the isomorphisms $\operatorname{Proj} R_{C} \cong \operatorname{Proj}\left(\bigoplus_{m \geq 0} H^{0}\left(C, \mathscr{O}\left(K_{C}\right)\right)\right) \cong C$ (where $K_{C} \sim$ $2(2 n-1) P$ is the canonical class of $C)$ show that if one requires that conditions (2.2.2) and (2.2.5) hold, then one can reconstruct the hyperelliptic curve $C$ of genus $2 n$ from the ring $R_{C}$.

\section{The canonical ring.}

3.1. Let $\sigma$ be the birational involution of the field $\mathbf{C}(S)$ over the field $\mathbf{C}(W)$. Since $S$ is a minimal surface we have that $\sigma$ is biregular and, moreover, $\sigma(P)=P$. Therefore the extension of $\sigma$ to $S^{\prime}$ is a biregular involution which preserves $E=$ $\pi^{-1}(P)$ and we denote it again by $\sigma$. The restriction of $\sigma$ on $L$ coincides with the hyperelliptic involution $i$ of this curve.

3.2. Now, we shall describe the canonical ring $R$ by generators and relations. The $i$-invariant degree 2 generator $z$ of the ring $R_{C}$ gives rise to a $\sigma$-invariant degree 2 generator $z$ of the ring $R$. We conclude from Theorem 2.2.9 that there exist $n-1$ relations

$$
D_{k}=h_{k} h_{1}^{2}-h_{k+1} z+h\left(e_{k+1} z+Q_{k}\right)=0, \quad k=1, \ldots, n-1,
$$

where $e_{k+1} \in \mathbf{C}$ and $Q_{k}$ are quadratic forms in $x$ and $y$. We rewrite these relations in the form $\left(h_{k+1}-e_{k+1} h\right) z=h_{k} h_{1}^{2}+h Q_{k}$ and lift them on $S^{\prime}$. Because of $P \notin(z)$ we have $h_{k+1}\left(x^{\prime}, y^{\prime}\right)-e_{k+1} h\left(x^{\prime}, y^{\prime}\right) \in H^{0}\left(S^{\prime}, \mathscr{O}(L-2 E)\right)$ (see 2.1. for notation) so that the line $l=F_{L}(E)$ divides the divisors $\left(h_{k+1}-e_{k+1} h\right)$ on $W=W_{c, d}$ for $k=1, \ldots, n-1$. Using Corollary 1.2 .7 and the notation from 1.3 we get $c_{k+1}(0,1)-e_{k+1} a(0,1)=0, d_{k+1}(0,1)-e_{k+1} b(0,1)=0, k=1, \ldots, n-1$, in Cases $\mathrm{A}$ and $\mathrm{C}$, and $c_{k+1}\left(z_{0}, z_{1}\right)-e_{k+1} a\left(z_{0}, z_{1}\right) \equiv 0, k=1, \ldots, n-1$, in Case B. In all cases we get $e_{k+1}=0, k=1, \ldots, n-1$, hence

$$
D_{k}=h_{k} h_{1}^{2}-h_{k+1} z+h Q_{k}=0, \quad k=1, \ldots, n-1 .
$$

Along the way we have proved

LEMMA 3.2.2. The line in $\mathbf{P}^{n}$ defined by the system $h_{2}=h_{3}=\cdots=h_{n}=0$ is exactly the line $l$ on $W$.

By analogy, the $i$-anti-invariant degree 3 generators $v_{1}, \ldots, v_{n-1}$ of the ring $R_{C}$ can be extended to $\sigma$-anti-invariant degree 3 generators, which we denote by the same letters, of the canonical ring $R$. Using again Theorem 2.2 .9 we have

$$
F_{p q}=v_{p} v_{q}-M_{p q}(x, y, z)=0, \quad 1 \leq p \leq q \leq n-1,
$$


where

$$
M_{p q}=\varphi_{[(p+q) / 2][(p+q+1) / 2]}\left(h_{1}^{2}, h_{1} h_{2}, \ldots, h_{n-1} h_{n}, h_{n}^{2}, z\right)+h \Phi_{p q}(x, y, z),
$$

$\Phi_{p q}$ are quasihomogeneous polynomials of degree 5 and they do not include $v_{p}$ because the other terms in the expressions above are $\sigma$-invariant;

$$
\begin{aligned}
G_{k s p q}=h_{k} v_{p}-h_{s} v_{q}+ & h \sum_{\lambda=1}^{n-1} g_{\lambda}^{(k s p q)} v_{\lambda}=0, \\
1 & \leq p<q \leq n-1,1 \leq k<s \leq n, s+p=k+q,
\end{aligned}
$$

where $g_{\lambda}^{(k s p q)} \in \mathbf{C}$

$$
\begin{aligned}
H_{p q}=z v_{p}-h_{[(q-p+1) / 2]} h_{[(q-p) / 2]+1} v_{q}+h \sum_{\lambda=1}^{n-1} h_{\lambda}^{(p q)} v_{\lambda} & =0 \\
1 & \leq p<q \leq n-1
\end{aligned}
$$

where $h_{\lambda}^{(p q)}$ are linear forms in $x$ and $y$.

We have proved the following

THEOREM 3.2.6. If $S$ is a minimal surface of general type with $K^{2}=2 p_{g}-3$, $p_{g} \geq 3$, such that the canonical linear system $|K|$ has one base point, and if $R$ is the canonical ring of $S$, then there is a natural isomorphism of graded rings $R=\mathbf{C}[x, y, z, v] / I$, where $\operatorname{deg} x_{i}=\operatorname{deg} y_{j}=1, \operatorname{deg} z=2, \operatorname{deg} v_{p}=3$ and the ideal $I$ is generated by the polynomials $a_{i j}, b_{l m}, c_{i m}$ (cf. (1.2.2), (1.2.3), (1.2.4)) of degree $2, D_{k}$ (cf. (3.2.1)) of degree $3, G_{k s p q}(c f .(3.2 .4))$ of degree 4, $H_{p q}(c f .(3.2 .5))$ of degree $5, F_{p q}(c f .(3.2 .3))$ of degree 6 .

\section{Close above the canonical image.}

4.1. A necessary and sufficient condition for equations (3.2.1) to be solvable with respect to $z$ is that the $(n-1)$-tuple $\left(Q_{1}, \ldots, Q_{n-1}\right)$ be a solution of the system

$$
h_{k+2}\left(h_{k} h_{1}^{2}+h Q_{k}\right)=h_{k+1}\left(h_{k+1} h_{1}^{2}+h Q_{k+1}\right), \quad k=1, \ldots, n-2,
$$

on the canonical image $W=W_{c, d}$. Using the parametrization (1.2.1) of the surface $W_{c, d}$ we have

$$
\begin{aligned}
h(x, y) & =\alpha a\left(z_{0}, z_{1}\right)+\beta b\left(z_{0}, z_{1}\right), \\
h_{k}(x, y) & =\alpha c_{k}\left(z_{0}, z_{1}\right)+\beta d_{k}\left(z_{0}, z_{1}\right), \quad k=1, \ldots, n,
\end{aligned}
$$

and (4.1.1) becomes equalities in the ring $\mathbf{C}\left[z_{0}, z_{1}, \alpha, \beta\right]$. If we rewrite them in the form $h\left(h_{k+2} Q_{k}-h_{k+1} Q_{k+1}\right)=h_{1}^{2}\left(h_{k+1}^{2}-h_{k} h_{k+2}\right)$, then we can use A.1.4, A.1.7, A.1.8 and get

$$
h_{k+2} Q_{k}-h_{k+1} Q_{k+1}=h_{1}^{2}\left(\alpha \zeta_{k}+\beta \delta_{k}\right), \quad k=1, \ldots, n-2 .
$$

We can suppose that $Q_{k}$ are quadratic forms of the variables

$$
h_{0}=h, h_{1}, \ldots, h_{n}: Q_{k}=\sum_{\lambda=0}^{n} \sum_{\mu=0}^{n} q_{\lambda \mu}^{(k)} h_{\lambda} h_{\mu},
$$


where $\left(q_{\lambda \mu}^{(k)}\right)$ are symmetric matrices. In Cases A and C equalities (A.1.5) show that $\left(\lambda_{1} h_{1}^{2}, \ldots, \lambda_{n-1} h_{1}^{2}\right)$ is a solution of the system (4.1.2). In Case B we use A.1.7 and the system (4.1.2) yields

$$
\begin{aligned}
& q_{00}^{(1)}=q_{00}^{(2)}=\cdots=q_{00}^{(n-2)}=0, \\
& q_{11}^{(2)}=-1 / b_{0} a_{1}, \quad q_{11}^{(3)}=\cdots=q_{11}^{(n-1)}=0, \\
& 2 q_{01}^{(k)}+b_{0} a_{1} q_{00}^{(k+1)}=0, \quad k=1, \ldots, n-2, \\
& q_{11}^{(k)}+2 b_{0} a_{1} q_{01}^{(k+1)}=0, \quad k=1, \ldots, n-2 .
\end{aligned}
$$

If $n>5$, then $q_{01}^{(3)}=1 / 2 b_{0}^{2} a_{1}^{2}$ and hence $0=q_{00}^{(4)}=-1 / b_{0}^{3} a_{1}^{3}$ : a contradiction. Therefore we have established the inequality $n \leq 5$, proved in $[5, \S 1]$. Conversely, if $3 \leq n \leq 5$, then using the relations (1.2.3) and (1.2.4) written in terms of the coordinates $h, h_{1}, \ldots, h_{n}$, we easily get that $\left(\bar{Q}_{1}, \ldots, \bar{Q}_{n-1}\right)$ is a solution of (4.1.2) where

$$
\begin{aligned}
& \bar{Q}_{1}=0 \\
& \bar{Q}_{2}=-\left(1 / b_{0} a_{1}\right) h_{1}^{2} \\
& \bar{Q}_{3}=\left(1 / b_{0}^{2} a_{1}^{2}\right) h h_{1}-\left(1 / b_{0} a_{1}\right) h_{1} h_{2}, \\
& \bar{Q}_{4}=-\left(1 / b_{0}^{3} a_{1}^{3}\right) h^{2}+\left(1 / b_{0}^{2} a_{1}^{2}\right) h h_{2}-\left(1 / b_{0} a_{1}\right) h_{1} h_{3} .
\end{aligned}
$$

4.2. Let $X=\operatorname{Proj} R$ be the canonical model of the surface $S$. Using Theorem 3.2.6 we get $X \subset \mathbf{P}^{2 n}\left(1^{n+1}, 2,3^{n-1}\right)$ and the surface $X$ is defined by equations (1.2.2)-(1.2.4), (3.2.1) and (3.2.3)-(3.2.5). The involution $\sigma$ (cf. 3.1) acts on the canonical ring $R$ by the rule $(x, y, z, v) \rightarrow(x, y, z,-v)$ and it is not hard to check that the ring $R^{\sigma}$ of invariants is isomorphic to $\mathrm{C}[x, y, z] / J$, where the ideal $J$ is generated by the polynomials $a_{i j}, b_{l m}, c_{i m}$ and $D_{k}$. Setting $V=\operatorname{Proj}\left(R^{\sigma}\right)$ we have $V \subset \mathbf{P}^{n+1}\left(1^{n+1}, 2\right)$ and the surface $V$ is defined by equations (1.2.2)(1.2.4) and (3.2.1); moreover, $V=X / \sigma$. The canonical map $F_{K}$ has the following decomposition:

$$
F_{K}: S \underset{M R}{\longrightarrow} X \underset{p r}{\longrightarrow} V \underset{p}{\rightarrow} W
$$

here $M R$ is the minimal resolution of the double rational points of $X, p r$ and $p$ are natural projections.

It follows from the equations of $X$ that the base point $P$ of the canonical linear system $|K|$ has coordinates $\left(0, \ldots, 0 ; 1 ; 0, \ldots, 0, e_{0}^{1 / 6}\right)$ on $X$. Note that $Q=$ $(0, \ldots, 0 ; 1)$ is the only point on $V$ at which the projection $p$ is not defined. By setting $x_{i}=X_{i}, y_{j}=Y_{j}, z=Z^{2}$ we lift the surface $V$ to a surface $V_{1}$ in the projective space $\mathbf{P}^{n+1}$ with coordinates $X_{i}, Y_{j}, Z$. If $\tau$ is the involution $\left(X_{i}, Y_{j}, Z\right) \rightarrow\left(X_{i}, Y_{j},-Z\right)$ of $V_{1}$, then $V=V_{1} / \tau$. Let us denote by $\eta$ the factor map $V_{1} \rightarrow V$ and by $p_{1}$ the natural projection $V_{1} \rightarrow W$. Then $p_{1}=p \circ \eta$ is not defined at the point $Q_{1}=\eta^{-1}(Q)$. The equations of $V_{1}$ show that every point on $W$ outside the line $l=\left(h_{2}=\cdots=h_{n}=0\right)$ (cf. Lemma 3.2.2) has, in general, two inverse images by $p_{1}$. The linear forms $h$ and $h_{1}$ are homogeneous coordinates on 
the line $l$ and a point $\left(h, h_{1}\right) \in l$ has an inverse image by $p_{1}$ if and only if

$$
\begin{aligned}
h^{3}+h Q\left(h, h_{1}, 0, \ldots, 0\right) & =0 \\
Q_{2}\left(h, h_{1}, 0, \ldots, 0\right) & =0 \\
\vdots & \\
Q_{n-1}\left(h, h_{1}, 0, \ldots, 0\right) & =0 .
\end{aligned}
$$

Set $f_{0}=f_{(0,1)}, f_{\infty}=f_{(1,0)}, c_{0}(x)=a(x), d_{0}(y)=b(y)$. It is clear that the coefficients of the linear forms $h_{k}(x, y)=c_{k}(x)+d_{k}(y), 0 \leq k \leq n$, are the cofactors of the elements of the $(k+1)$ st column of the matrix (1.3.1) (in Cases A and C), respectively of the matrix (1.3.2) (in Case B), and the multiplicity of the line $f_{0}$ (respectively, the line $f_{\infty}$ ) in the divisor $\left(h_{k}\right)$ on $W_{c, d}$ is just the minimum of the multiplicities of $z_{0}$ (respectively, of $z_{1}$ ) in the polynomials $c_{k}\left(z_{0}, z_{1}\right), d_{k}\left(z_{0}, z_{1}\right)$. In Case A our choice of the first column of the matrix (1.3.1) and Lemma A.1.2, $1^{\circ}$ show that the minimum of the multiplicities of $z_{0}$ is $\geq 1$ for $2 \leq k \leq n$. On the other hand for a generic hyperplane section $h(x, y)$ all cofactors of the elements of the second, the $(c+1)$ st, the $(c+3)$ rd, and the last row and the $k$ th column of the matrix (1.3.1), $2 \leq k \leq n$, are $\neq 0$, so, in particular, this minimum is equal to 1 . Moreover, the line $f_{\infty}$ does not divide $\left(h_{k}\right), 2 \leq k \leq n$.

In Cases $\mathrm{B}$ and $\mathrm{C}$ we have for $k=2, \ldots, n$ :

$$
\begin{aligned}
& c_{k}\left(z_{0}, z_{1}\right) \equiv 0, \\
& d_{k}\left(z_{0}, z_{1}\right)= \begin{cases}\left(1 / a_{1}\right) z_{0}^{k-2} z_{1}^{n-k} & \text { in Case B, } \\
\left(1 / a_{0}\right) z_{0}^{k-1} z_{1}^{n-k} & \text { in Case C. }\end{cases}
\end{aligned}
$$

We have proved the following

LEMMA 4.2.2. For a generic hyperplane section $h(x, y)$ one has

$$
\left(h_{k}\right)= \begin{cases}H_{k}+l, & \text { in Case A, } \\ l+(k-2) f_{0}+(n-k) f_{\infty}, & \text { in Case B, } \\ (k-1) f_{0}+(n-k) f_{\infty}, & \text { in Case C, } \quad k=2, \ldots, n,\end{cases}
$$

where $H_{k}$ is a rational curve on $W_{c, d}$ and $\left(H_{k}, f\right)=1$.

Let us rewrite (4.1.1) in the form

$$
\left(h_{k+2} / h_{2}\right)\left(h_{1}^{3}+h Q_{1}\right)=h_{k+1} h_{1}^{2}+h Q_{k+1}, \quad k=1, \ldots, n-2 .
$$

Lemma 4.2.2 shows that in Cases A and B the restriction of the rational function $h_{k+2} / h_{2}$ on the line $l$ is a nonzero rational function $g_{k+1}$ with divisor

$$
\left(g_{k+1}\right)= \begin{cases}P_{k+2}-P_{2}, & \text { in Case A, } \\ k P_{0}-k P_{\infty}, & \text { in Case B, }\end{cases}
$$

where $P_{k}=H_{k} \cap l, k=2, \ldots, n$, in Case A, and $P_{0}=f_{0} \cap E_{\infty}, P_{\infty}=f_{\infty} \cap E_{\infty}$. In Case $\mathrm{C}$ the function $g_{k+1}$ is identically zero on the line $l$ for $k=1, \ldots, n-2$. We have $g_{k+1}\left(h_{1}^{3}+h Q_{1}\right)=h Q_{k+1}$ on $l$, hence $Q_{k+1}=0$ in Case C and $\left(g_{k+1}\right)+\left(h_{1}^{3}+h Q_{1}\right)=$ $(h)+\left(Q_{k+1}\right)$ in the other cases for $k=1, \ldots, n-2$.

Let us first consider Case C. Then the system (4.2.1) is equivalent to its first equation; the vertex $E_{\infty}$ of the cone $W_{0, n-1}$ has coordinates $\left(h, h_{1}\right)=\left(a_{0},-1 / b_{0}\right)$ 
and because of (4.1.2) it satisfies this equation. Hence $\left(h_{1}^{3}+h Q_{1}\right)=T_{0}+T_{1}+T_{2}$ where $T_{0}=E_{\infty}$ and $\left\{T_{0}, T_{1}, T_{2}\right\}$ is the set of solutions of (4.2.1) in this case.

In Case A we have $P_{k+2}-P_{2}+\left(h_{1}^{3}+h Q_{1}\right)=P_{h}+\left(Q_{k+1}\right)$ and because of $P_{h} \nmid\left(h_{1}^{3}+h Q_{1}\right)$ we get $P_{k+2} \neq P_{2}, P_{k+2}=P_{h}, P_{2} \mid\left(h_{1}^{3}+h Q_{1}\right)$ and $\left(Q_{k+1}\right)=$ $\left(h_{1}^{3}+h Q_{1}\right)-P_{2}$, for all $k=1, \ldots, n-2$. If $\left(h_{1}^{3}+h Q_{1}\right)=P_{2}+T_{1}+T_{2}$, then in this case $\left\{T_{1}, T_{2}\right\}$ is the set of solutions of (4.2.1).

In Case B we have $3 \leq n \leq 5, P_{h}=P_{0}$ and $\left(Q_{k+1}\right)=(k-1) P_{0}-k P_{\infty}+\left(h_{1}^{3}+h Q_{1}\right)$ for $k=1, \ldots, n-2$; hence $(n-2) P_{\infty} \mid\left(h_{1}^{3}+h Q_{1}\right)$ and if $\left(h_{1}^{3}+h Q_{1}\right)=(n-2) P_{\infty}+$ $T_{1}+\cdots+T_{5-n}$, then

$$
\left(Q_{k+1}\right)=(k-1) P_{0}+(n-2-k) P_{\infty}+T_{1}+\cdots+T_{5-n}, \quad k=1, \ldots, n-2 .
$$

Therefore $\left\{T_{1}, \ldots, T_{5-n}\right\}$ is the set of solutions of (4.2.1) in Case B.

4.3. The Jacobian of the system which defines the surface $V_{1}$ shows that the plane $\left(Q_{1}, l\right)$ is the tangential space to $V_{1}$ at the point $Q_{1}$, hence $V_{1}$ is smooth at $Q_{1}$ and the functions $h / Z, h_{1} / Z$ are local parameters at this point. Because of $\tau(h / Z)=-h / Z, \tau\left(h_{1} / Z\right)=-h_{1} / Z$ the point $Q=\eta\left(Q_{1}\right)$ is a double rational point of type $A_{1}$ on the surface $V$. Let $q_{1}: V_{1}^{\prime} \rightarrow V_{1}$ be the monoidal transformation with center $Q_{1}$ and exceptional curve $G_{1}$. It is clear that $q_{1}$ resolves the irregular point $Q_{1}$ of the projection $p_{1}$. The involution $\tau$ of $V_{1}$ can be extended to an involution $\tau$ of $V_{1}^{\prime}$ which preserves the curve $G_{1}$. We denote by $V^{\prime}$ the factor variety $V_{1}^{\prime} / \tau$ and by $\eta^{\prime}$ the factor map $V_{1}^{\prime} \rightarrow V^{\prime}$. The image $\eta^{\prime}\left(G_{1}\right)=G$ is a rational curve and $\eta^{\prime *}(G)=2 G_{1}$. We have $2 G^{2}=4 G_{1}^{2}=-4$, hence $G^{2}=-2$. Since $V_{1}^{\prime}-G_{1}$ is isomorphic to $V_{1}-Q_{1}$, then $V^{\prime}-G$ is isomorphic to $V-Q$, so the natural map $q: V^{\prime} \rightarrow V$ is the minimal resolution of the double rational point $Q \in V$.

Let $p_{1}^{\prime}$ be the regular extension of $p_{1}$ on $V_{1}^{\prime}$. The map $p_{1}^{\prime}$ is $\tau$-equivariant and the factor map $p^{\prime}: V^{\prime} \rightarrow W$ is regular and an isomorphism outside the inverse images of the points $T_{\mu}$ which form the set of solutions of (4.2.1). It is not hard to see that, in fact, $p^{\prime}$ is the composition of the monoidal transformations with centers $T_{\mu}$ (the points $T_{\mu}$ may be infinitely near). Denote by $\Theta_{\mu}$ their exceptional lines.

Let $\rho: X^{\prime} \rightarrow X$ be the monoidal transformation with center $P$ and exceptional line $G^{\prime}$ and let $\varphi: S^{\prime} \rightarrow X^{\prime}$ and $\psi: X^{\prime} \rightarrow V^{\prime}$ be the extensions of the maps $M R$ and $p r$ respectively. We have constructed the following commutative diagram:

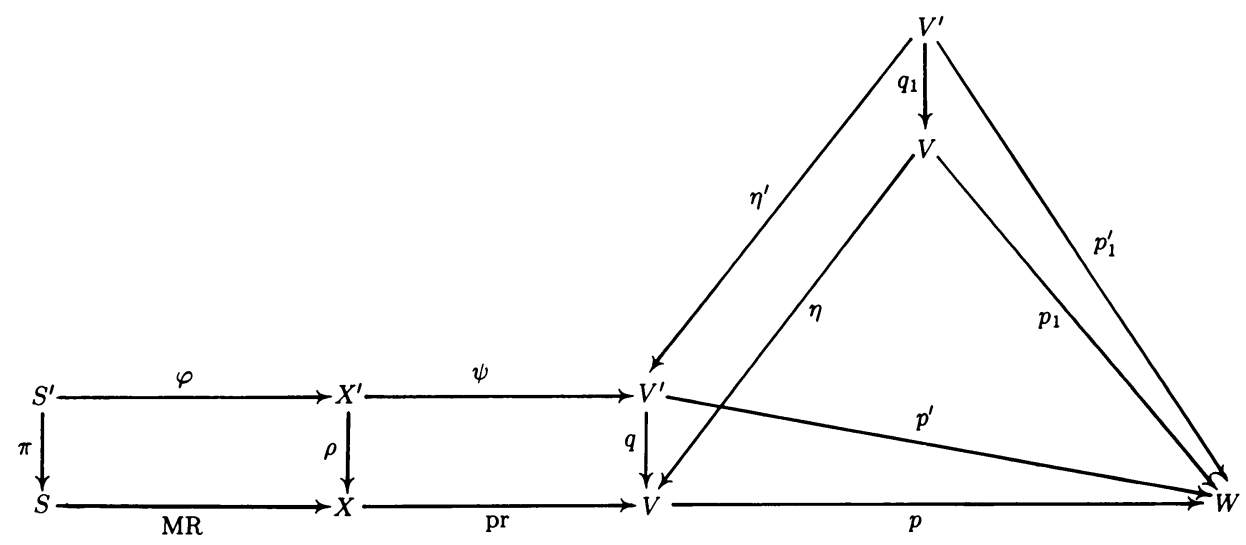




\section{Characterization theorem.}

5.1. The following equalities give necessary and sufficient conditions for solving (3.2.3) on the surface $V$ with respect to $v_{1}, \ldots, v_{n-1}$ :

$$
M_{p q} M_{r s}=M_{p r} M_{q s}, \quad 1 \leq p, q, r, s \leq n-1, \text { on } V
$$

(we set $M_{q p}=M_{p q}$ ). If they hold, (3.2.3) has two solutions: $(x, y, z, v)$ and $(x, y, z,-v)=\sigma(x, y, z, v)$ which form the canonical model $X$ of the surface $S$ when $(x, y, z)$ varies on $V$.

The branch locus $B$ of the 2-1 map $p r$ is defined by the equations $M_{p q}=0$, $1 \leq p \leq q \leq n-1$, on the surface $V$. Because of $0 \neq e_{0}=M_{n-1, n-1}(Q)$ (cf. (2.2.2)) we have $Q \notin B$, hence $\left(q^{*} B, G\right)=0$ and without ambiguity we may denote $q^{*} B$ by $B$. Since $\psi^{*}(G)=2 G^{\prime}$, the branch locus of the $2-1$ map $\psi$ is $B+G$.

Suppose we are in Case A or Case B. In [4 and 5] Horikawa computes the branch loci of the compositions $\chi=\psi \circ \varphi$ and $F_{L}=p^{\prime} \circ \chi$ as follows: the branch locus of $F_{L}$ is in the linear system

$$
\left|2(3+\varepsilon) E_{\infty}+(n+5+3 d-2 \varepsilon) f\right| \text { on } W ;
$$

the branch locus of $\chi$ is in the linear system

$$
\left|2(3+\varepsilon) E_{\infty}+(n+5+3 d-2 \varepsilon) f-4 \sum_{\mu} \Theta_{\mu}\right| \quad \text { on } V^{\prime} .
$$

The fact that $X$ has at most double rational points as singularities yields that the branch locus $B+G$ of $\chi$ has neither multiple components nor infinitely near triple points (cf. [4, Lemma 5]); moreover, $d \leq(n+2) / 2$. Conversely, if this inequality is satisfied, then under some locally closed conditions (called Conditions $B_{d}$ ) we have:

$1^{\circ}$. There exists a not empty open set of curves in (5.1.2) which have neither multiple components nor infinitely near triple points (cf. [5, Lemma 1.2.]).

$2^{\circ}$. The branch curve $B$ of $p r$ is such that $B+G$ on $V^{\prime}$ coincides with a curve from the open set defined in $1^{\circ}$.

Then, supposing (5.1.1) and all conditions which assure the existence of the surface $V$ (see the previous section) we infer that the surface $X_{1}$ defined by the ideal $I_{1}=\left(a_{i j}, b_{l m}, c_{i m}, D_{k}, F_{p q}\right)$ is a 2-1 covering of $V$ ramified over the curve $B$ and over the unique singular point $Q$ on $V$. Hence the blow-up $X_{1}^{\prime}$ of $X_{1}$ at the point $P$ is a 2-1 covering of $V^{\prime}$ ramified over the curve $B+G$. Both $X_{1}$ and $X_{1}^{\prime}$ have at most double rational points as singularities, the minimal resolution $S_{1}^{\prime}$ of $X_{1}^{\prime}$ is the blow-up of the minimal resolution $S_{1}$ of $X_{1}$ at the point $P$, the last surface $S_{1}$ is a minimal surface of general type with $p_{g}=n+1, K^{2}=2 n-1$, such that its canonical linear system defines the natural map onto $W$ with unique base point $P$ (cf. [4 and 5$]$ ).

We obtain from (3.2.4) and (3.2.5) a necessary condition for the coincidence of $X$ and $X_{1}$ (and, hence, of $S$ and $S_{1}$ ): there exist constants $g_{\lambda}^{(k s p q)}$ and linear forms $h_{\lambda}^{(p q)}$ in $x$ and $y$ such that

$$
\begin{aligned}
& h_{k} M_{p r}-h_{s} M_{q r}+h \sum_{\lambda=1}^{n-1} g_{\lambda}^{(k s p q)} M_{\lambda r}=0, \quad 1 \leq p<q \leq n-1, \\
& 1 \leq k<s \leq n, s+p=k+q, 1 \leq r \leq n-1,
\end{aligned}
$$




$$
\begin{aligned}
& z M_{p r}-h_{[(q-p+1) / 2]} h_{[(q-p) / 2]+1} M_{q r}+h \sum_{\lambda=1}^{n-1} h_{\lambda}^{(p q)} M_{\lambda r}=0, \\
& 1 \leq p<q \leq n-1,1 \leq r \leq n-1,
\end{aligned}
$$

on $V$. Hence we have the syzygies

$$
\begin{gathered}
v_{r} G_{k s p q}-h_{k} F_{p r}+h_{s} F_{q r}-h \sum_{\lambda=1}^{n-1} g_{\lambda}^{(k s p q)} F_{\lambda r}=0 \\
v_{r} H_{p q}-z F_{p r}+h_{[(q-p+1) / 2]} h_{[(q-p) / 2]+1} F_{q r}-h \sum_{\lambda=1}^{n-1} h_{\lambda}^{(p q)} F_{\lambda r}=0
\end{gathered}
$$

where the indices run over the same values as (5.1.3) and (5.1.4) (compare with (2.2.11) and (2.2.12)). Conversely, supposing that (5.1.3) and (5.1.4) hold and using the syzygies (5.1.5) and (5.1.6) we infer that $\operatorname{rad} I_{1}=\operatorname{rad} I$, hence $X_{1}=X$.

5.2. Now, let us suppose that we are in Case C. With an abuse of notation we denote by $E_{\infty}$ the exceptional line $\Theta_{0}$ of the monoidal transformation $m_{0}$ with center at the vertex $E_{\infty}=T_{0}$ of the cone $W_{0, n-1}$. The result of this monoidal transformation is a ruled surface $W_{n-1}$ with directrix $E_{\infty}, E_{\infty}^{2}=-n+1$, a zero section $E_{0}$ and such that its fibres are exactly the proper transforms of the rulings of the cone. The surface $V^{\prime}$ is obtained by applying two monoidal transformations $m_{1}$ and $m_{2}$ with centers at the points $T_{1}$ and $T_{2}$ on the fibre $l$ of $W_{n-1}(l$ is the proper transform of the line $l$ on the cone $\left.W_{0, n-1}\right)$.

Let us suppose $W_{n-1}$ is embedded in $\mathbf{P}^{n+2}$ by the linear system $\left|E_{\infty}+n f\right|$ as a rational normal scroll $W_{1, n-1}$. Set $\chi_{1}=m_{1} \circ m_{2} \circ \chi$ and $F=\chi_{1}^{*}(f)$. We have $L=\chi_{1}^{*}\left(E_{0}\right), F_{L}=m_{0} \circ \chi_{1}$, and using the long exact sequence

$$
0 \rightarrow H^{0}\left(S^{\prime}, \mathscr{O}(L)\right) \rightarrow H^{0}\left(S^{\prime}, \mathscr{O}(L+F)\right) \rightarrow H^{0}\left(F, \mathscr{O}\left(K_{F}\right)\right) \rightarrow \cdots
$$

it is easy to see that $\operatorname{dim} H^{0}\left(S^{\prime}, \mathscr{O}(L+F)\right)=n+3$ and $\chi_{1}$ and $F_{L}$ can be defined by choosing a basis of this $\mathbf{C}$-linear space in such a way that the monoidal transformation $m_{0}: W_{1, n-1} \rightarrow W_{0, n-1}$ is the restriction of the projection

$$
\begin{aligned}
& \mathbf{P}^{n+2} \rightarrow \mathbf{P}^{n}, \\
& \left(x_{0}, x_{1} ; y_{0}, y_{1}, \ldots, y_{n-1}, y_{n}\right) \mapsto\left(x_{0} ; y_{0}, \ldots, y_{n-1}\right) .
\end{aligned}
$$

Horikawa shows in [5] that the branch loci of $\chi_{1}$ and $\chi$ belong to the linear systems $\left|6 E_{\infty}+(4 n+2) f\right|$ on $W_{1, n-1}$ and $\left|6 E_{\infty}+(4 n+2)-4 \sum_{\lambda=1}^{2} \Theta_{\lambda}\right|$ on $V^{\prime}$, respectively, that the branch locus of $\chi$ has neither multiple components nor infinitely near triple points, and he reduced Case $\mathrm{C}$ to Case A with $d=n-1$. More precisely, all conditions in 5.1 concerning Case A with $d=n-1$ hold in Case $\mathrm{C}$ after replacing $F_{L}$ with $\chi_{1}$.

5.3. For the convenience of the reader we collect in the list below all conditions which the parameters of the relations of the canonical ring $R$ satisfy.

$$
\begin{aligned}
& 2 \leq n, 0 \leq c, 0 \leq d \leq(n+2) / 2,2 c+d=n-1 \\
& c>0, \varepsilon=0 \text { in Case A; } \\
& c=1,3 \leq n \leq 5, \varepsilon=1 \quad \text { in Case } \mathrm{B} \\
& c=0,2 \leq n \leq 4 \quad \text { in Case C. }
\end{aligned}
$$


The point $\left(a_{0}, \ldots, a_{c} ; b_{0}, \ldots, b_{c+d}\right) \in\left(\mathbf{P}^{n}\right)^{*}$ is in sufficiently general position; $a_{0}=0$ in Case B (on account of choice of a coordinate system in $\mathbf{P}^{n}$, see (2.1.1)). The polynomial $f_{4 n+1}\left(z_{0}, z_{1}\right)=\sum_{\nu=0}^{4 n+1} e_{\nu} z_{0}^{\nu} z_{1}^{4 n+1-\nu}$ has simple roots and $e_{0} \neq 0$ (cf. $(2.2 .2))$.

$$
\begin{aligned}
& \varphi_{[(p+q) / 2][(p+q+1) / 2]}\left(z_{0} z_{1}^{2 n-2}, z_{0}^{2} z_{1}^{2 n-3}, \ldots, z_{0}^{2 n-1}, z_{1}^{2 n-1}\right) \\
& =z_{0}^{2 n-p-q-2} z_{1}^{p+q+2} f_{4 n+1}\left(z_{0}, z_{1}\right), \quad 1 \leq p \leq q \leq n-1 \\
& h_{k+2}\left(h_{k} h_{1}^{2}+h Q_{k}\right)=h_{k+1}\left(h_{k+1} h_{1}^{2}+h Q_{k+1}\right), \quad k=1, \ldots, n-2, \text { on } W \\
& \text { (cf. (4.1.1)); } \\
& M_{p q} M_{r s}=M_{p r} M_{q s}, \quad 1 \leq p, q, r, s \leq n-1, \text { on } V \quad \text { (cf. (5.1.1)); } \\
& h_{k} M_{p r}-h_{s} M_{q r}+h \sum_{\lambda=1}^{n-1} g_{\lambda}^{(k s p q)} M_{\lambda r}=0, \quad 1 \leq p<q \leq n-1,1 \leq k<s \leq n \\
& s+p=k+q, 1 \leq r \leq n-1 \text {, on } V \\
& \text { (cf. (5.1.3)); } \\
& z M_{p r}-h_{[(q-p+1) / 2]} h_{[(q-p) / 2]+1} M_{q r}+h \sum_{\lambda=1}^{n-1} h_{\lambda}^{(p q)} M_{\lambda r}=0 \\
& 1 \leq p<q \leq n-1,1 \leq r \leq n-1 \text {, on } V \quad \text { (cf. (5.1.4)). }
\end{aligned}
$$

The polynomials $M_{p q}, 1 \leq p \leq q \leq n-1$, and $Q_{k}, 1 \leq k \leq n-1$, satisfy Conditions $B_{d}$ from 5.1.

THEOREM 5.3.2. Let $R$ be the ring $\mathbf{C}[x, y, z, v] / I$ from Theorem 3.2 .6 where the ideal I satisfies the conditions from (5.3.1). Then $X=\operatorname{Proj} R$ is a surface which has at most double rational points as singularities. The minimal resolution $S$ of $X$ is a minimal surface of general type with $p_{g}=n+1, K^{2}=2 n-1$, and such that its canonical linear system has one base point. The ring $R$ is naturally isomorphic to the canonical ring of $S$.

PROOF. The proof of the theorem has been completed before its formulation. Table 1 classifies these surfaces.

\begin{tabular}{ccccll}
\hline$p_{g}$ & $K^{2}$ & $c$ & $d$ & $\begin{array}{c}\text { The canonical } \\
\text { image }\end{array}$ & Type \\
\hline 3 & 3 & 0 & 1 & $W_{0,1} \cong \mathbf{P}^{2}$ & Case C \\
4 & 5 & 0 & 2 & $W_{0,2}$ & Case C \\
4 & 5 & 1 & 0 & $W_{1,0}=\mathbf{P}^{1} \times \mathbf{P}^{1}$ & Case A=Case B \\
5 & 7 & 0 & 3 & $W_{0,3}$ & Case C \\
5 & 7 & 1 & 1 & $W_{1,1}$ & Case A and Case B \\
6 & 9 & 1 & 2 & $W_{1,2}$ & Case A and Case B \\
6 & 9 & 2 & 0 & $W_{2,0}$ & Case A \\
$\geq 7$ & & $>0$ & $\leq\left(p_{g}+1\right) / 2$ & $W_{c, d}$, & Case A \\
& & & & $2 c+d=p_{g}-2$ & \\
\hline
\end{tabular}




\section{Appendix A.}

A.1. Set $z=\left(z_{0}, z_{1}\right)$ and let $a(z)=a_{0} z_{1}^{c}+a_{1} z_{0} z_{1}^{c-1}+\cdots+a_{c} z_{0}^{c}, b(z)=$ $b_{0} z_{1}^{c+d}+b_{1} z_{0} z_{1}^{c+d-1}+\cdots+b_{c+d} z_{0}^{c+d}$ be mutually prime homogeneous polynomials, $2 c+d=n-1, n \geq 2$. Then for every $k, 1 \leq k \leq n$, there exists a pair $c_{k}(z), d_{k}(z)$ of homogeneous polynomials of degrees $c$ and $c+d$ respectively, such that

$$
a(z) d_{k}(z)-b(z) c_{k}(z)=z_{0}^{k-1} z_{1}^{n-k}
$$

furthermore, any pair $c_{k}^{\prime}(z), d_{k}^{\prime}(z)$ with this property is given by the formulae

$$
c_{k}^{\prime}(z)=c_{k}(z)+\lambda_{k} a(z), \quad d_{k}^{\prime}(z)=d_{k}(z)+\lambda_{k} b(z)
$$

for some constants $\lambda_{k}(1 \leq k \leq n)$.

The proof of the following lemma is obvious.

LEMMA A.1.2. $1^{\circ}$. If $k>1$ and $a_{0} \neq 0\left(b_{0} \neq 0\right)$, then there exists a single pair $c_{k}(z), d_{k}(z)$ which satisfies $(\mathrm{A} .1 .1)$ and such that $c_{k}(0,1)=0\left(d_{k}(0,1)=0\right)$. This yields $d_{k}(0,1)=0\left(c_{k}(0,1)=0\right)$.

$2^{\circ}$. If $k=1$ and $a_{0} \neq 0\left(b_{0} \neq 0\right)$, then there exists a single pair $c_{1}(z), d_{1}(z)$ which satisfies (A.1.1) and such that $c_{1}(0,1)=0\left(d_{1}(0,1)=0\right)$. This yields $d_{1}(0,1) \neq 0$ $\left(c_{1}(0,1) \neq 0\right)$.

$3^{\circ}$. If $k<n$ and $a_{c} \neq 0\left(b_{c+d} \neq 0\right)$, then there exists a single pair $c_{k}(z)$, $d_{k}(z)$ which satisfies (A.1.1) and such that $c_{k}(1,0)=0\left(d_{k}(1,0)=0\right)$. This yields $d_{k}(1,0)=0\left(c_{k}(1,0)=0\right)$.

$4^{\circ}$. If $c_{k}(z) \equiv 0$ for some $k, 1 \leq k \leq n$, then $a(z)=a_{i} z_{0}^{i} z_{1}^{c-i}$ with $0 \leq i \leq n-1$, $0 \leq c-i \leq n-k$ and $d_{k}(z)=z_{0}^{k-1} z_{1}^{n-k} / a(z)$. In particular, if $c_{k}(z) \equiv 0$ for $k=2, \ldots, n$, then $i=c=0$ or 1 .

Let $c_{k}(z), d_{k}(z)$ be the pairs from $1^{\circ}$ and $2^{\circ}$ of Lemma A.1.2. Then for every $k=1, \ldots, n-1$, we have

$$
a(z) d_{k+1}(z)\left(z_{1} / z_{0}\right)-b(z) c_{k+1}(z)\left(z_{1} / z_{0}\right)=z_{0}^{k-1} z_{1}^{n-k}
$$

hence there exist constants $\lambda_{k}$ such that

$$
\begin{aligned}
& c_{k+1}(z)\left(z_{1} / z_{0}\right)=c_{k}(z)+\lambda_{k} a(z), \\
& d_{k+1}(z)\left(z_{1} / z_{0}\right)=d_{k}(z)+\lambda_{k} b(z) .
\end{aligned}
$$

Now, we shall be dealing with the expression

$$
\begin{aligned}
A_{k}(z ; \alpha, \beta)= & \left(\alpha c_{k+1}(z)+\beta d_{k+1}(z)\right)^{2} \\
& -\left(\alpha c_{k}(z)+\beta d_{k}(z)\right)\left(\alpha c_{k+2}(z)+\beta d_{k+2}(z)\right) .
\end{aligned}
$$

LEMMA A.1.4. One has

$$
A_{k}(z ; \alpha, \beta)=\left(\alpha \varsigma_{k}(z)+\beta \delta_{k}(z)\right)(\alpha a(z)+\beta b(z)), \quad k=1, \ldots, n-2,
$$

where

$$
\begin{aligned}
& \varsigma_{k}(z)=\lambda_{k} c_{k+2}(z)-\lambda_{k+1} c_{k+1}(z), \\
& \delta_{k}(z)=\lambda_{k} d_{k+2}(z)-\lambda_{k+1} d_{k+1}(z) .
\end{aligned}
$$

PROOF. Because of $A_{k}(z ;-b(z), a(z)) \equiv 0$ we can find homogeneous polynomials $\varsigma_{k}(z)$ and $\delta_{k}(z)$ of degrees $c$ and $c+d$ respectively, such that

$$
\begin{aligned}
& c_{k+1}^{2}(z)-c_{k}(z) c_{k+2}(z)=a(z) \zeta_{k}(z), \\
& d_{k+1}^{2}(z)-d_{k}(z) d_{k+2}(z)=b(z) \delta_{k}(z) .
\end{aligned}
$$


This yields

$$
2 c_{k+1}(z) d_{k+1}(z)-c_{k}(z) d_{k+2}(z)-c_{k+2}(z) d_{k}(z)=b(z) \zeta_{k}(z)+a(z) \delta_{k}(z)
$$

and we get the decomposition of $A_{k}(z ; \alpha, \beta)$. Multiplying (A.1.6) by $z_{0}$ and using (A.1.3) we have

$$
\begin{aligned}
z_{0} a(z) \zeta_{k}(z)= & c_{k+1}(z)\left(z_{1} c_{k+2}(z)-z_{0} \lambda_{k+1} a(z)\right) \\
& -c_{k+2}(z)\left(z_{1} c_{k+1}(z)-z_{0} \lambda_{k} a(z)\right) \\
= & z_{0} a(z)\left(\lambda_{k} c_{k+2}(z)-\lambda_{k+1} c_{k+1}(z)\right),
\end{aligned}
$$

hence we have the first equality from (A.1.5). The proof of the second equality can be done in a similar way.

REMARK A.1.7. Suppose $c=1$. If $c_{k}(z), d_{k}(z), k=2, \ldots, n$, are the pairs from $4^{\circ}$ of Lemma A.1.2 and $c_{1}(z), d_{1}(z)$ is the pair from $3^{\circ}$ of the same lemma (that is $\left.c_{1}(z)=-\left(1 / b_{0}\right) z_{1}, b_{0} a_{1} z_{0} d_{1}(z)=b_{0} z_{1}^{n-1}-b(z) z_{1}\right)$, then

$$
A_{k}(z ; \alpha, \beta)=\left(\alpha \zeta_{k}(z)+\beta \delta_{k}(z)\right)(\alpha a(z)+\beta b(z)),
$$

where $\zeta_{k}(z)=\delta_{k}(z)=0$ for $k=2, \ldots, n$, and $\zeta_{1}(z)=0, \delta_{1}(z)=\left(1 / b_{0} a_{1}^{2}\right) z_{1}^{n-2}$.

REMARK A.1.8. Suppose $c=0$. If $c_{k}(z), d_{k}(z), k=2, \ldots, n$, are the pairs from $4^{\circ}$ of Lemma A.1.2 and $c_{1}(z), d_{1}(z)$ is the pair from $2^{\circ}$ of the same lemma (that is $\left.c_{1}(z)=-1 / b_{0}, a_{0} d_{1}(z)=z_{1}^{n-1}-\left(1 / b_{0}\right) b(z)\right)$, then

$$
A_{k}(z ; \alpha, \beta)=\left(\alpha \zeta_{k}(z)+\beta \delta_{k}(z)\right)(\alpha a(z)+\beta b(z)),
$$

where $\varsigma_{k}(z)=\delta_{k}(z)=0$ for $k=2, \ldots, n$, and $\varsigma_{1}(z)=0, \delta_{1}(z)=\left(1 / b_{0} a_{0}^{2}\right) z_{0}^{2} z_{1}^{n-3}$.

\section{REFERENCES}

1. E. Bombieri, Canonical models of surfaces of general type, Inst. Hautes Études Sci. Publ. Math. 42 (1973), 179-219.

2. E. Griffin, Special fibres in families of plane curves, Ph.D. thesis, Harvard Univ., 1982.

3. Ph. Griffiths and J. Harris, Principles of algebraic geometry, Pure and Applied Math., Wiley, 1978.

4. E. Horikawa, On deformations of quintic surfaces, Invent. Math. 31 (1975), 43-85.

5. $\ldots$, Algebraic surfaces of general type with small $c_{1}^{2}$. II, Invent Math. 37 (1976), 121-155.

6. V. Iliev, A note on certain surfaces, Bull. London Math. Soc. 16 (1984), 135-138.

7. K. Kodaira, Pluricanonical systems on algebraic surfaces of general type, J. Math. Soc. Japan 20 (1968), 170-192.

Institute of Mathematics, Bulgarian ACAdemy of Sciences, Sofia, Bulgaria 